\title{
EVALUATING DIFFERENT DISPLAY TECHNIQUES FOR COMMUNICATING SEARCH STRATEGY TRAINING IN A COLLABORATIVE VIRTUAL AIRCRAFT INSPECTION ENVIRONMENT.
}

\author{
P. Mehta, S. Sadasivan, J. S. Greenstein, and A. K. Gramopadhye \\ Department of Industrial Engineering \\ A. T. Duchowski \\ Department of Computer Science \\ Clemson University, Clemson, SC 29634, USA
}

\begin{abstract}
Aircraft inspection is vital to assure safe and reliable air transportation. Search strategy training has been recognized to be effective in improving an inspector's performance in a visual inspection task. Improving the search performance of novice inspectors can be expedited by providing cognitive feedfoward information about the search strategy adopted by an expert inspector. In a collaborative virtual aircraft inspection environment using eye tracking equipment, novice inspectors can observe in real time the point of regard (POR) data of an expert inspector performing an inspection task. This research evaluates the effectiveness of three display techniques -'dot', 'ray casting' and 'decaying trace' -- for representing, the gaze slaved visual deictic reference (VDR) of the expert inspector during search strategy training in an aircraft inspection task. Increase in performance of the novices performing an inspection task after training show the 'decaying trace' as the most effective form for representing the expert's VDR.
\end{abstract}

\section{INTRODUCTION}

Research investigating aircraft inspection procedures remains an important focus, especially in light of the fact that approximately $18 \%$ of all major aircraft accidents have been attributed to maintenance and inspection errors. Aircraft inspection and maintenance tends to be a visual process conducted by human inspectors searching the airframe structure for defects. Of particular importance are locating and marking indications of defects like cracks and corrosion. Training has been identified as the primary intervention for enhancing inspection performance (Drury and Gramopadhye, 1990). Adopting a systematic search strategy has been widely recognized as valuable in improving the visual search behavior. Current aircraft inspection training tends to be primarily on-the-job training (OJT). However, OJT has its disadvantages including delayed feedback and limited exposure to different airframe structures and defects. PC based training simulators were explored to remedy this but they do not provide a holistic view of airframe structures. A disadvantage addressed by the Virtual Reality (VR) aircraft inspection simulator (Duchowski et al., 2000) developed at Clemson University. Previous studies using the VR training simulator have been shown to be effective in improving inspection performance but it lacks the one on one interaction provided by OJT. A collaborative virtual environment (CVE) enhances the current single-user VR technology by allowing for the co-immersion of both the expert and the novice inspector thus simulating OJT. The key to the success of training using a CVE is the ability of the participants to interpret and monitor each other's conduct while performing an inspection task. Early research suggests that the presence of an animated character, i.e. an avatar, even with rather primitive expressiveness, representing the position 
and orientation of the co-participants in a CVE facilitates collaboration thereby enhancing both learning and motivation. The avatars used to depict participants in the CVE at Clemson are capable of torso movements along with head and eye rotations for effective collaboration.

In the real world, humans most commonly use the pointing gesture, sometimes accompanied by a verbal cue such as "look at this" or "see that," to mark a specific object, location or direction. This is known as his deictic reference (Hindmarsh et al., 2000). Using a visual aid may be the most effective method as exemplified by the GAZE Groupware System (Vertegaal, 1999) where the gaze direction of the participants collaborating on a document in a virtual environment was represented in the form of an animated colored dot. Visual deictic reference (VDR) of the expert's area of interest could be derived from: the head orientation tracked by the position of the Head Mounted Display (HMD); gaze tracking using a binocular eye-tracker; or from the orientation of a 3D wand used for pointing.

Previous studies have shown providing an eye position feedback aid for the inspection of the chest radiographs increases accuracy significantly (Kundel et al., 1990). Providing recorded eye movements as feedforward information has shown to improve the visual search behavior of inspectors, especially novice ones. Comparing the eye movement strategies adopted by experts and novices in a virtual training task can be used to diferentiate the novice from the expert (Law et al. 2004). This research explores the use of VDR slaved to the gaze orientation of an expert, while evaluating the effectiveness of three display techniques to represent the same--a dot, ray casting and a decaying trace-for search strategy training in a collaborative aircraft inspection environment.

\section{EXPERIMENTAL SETUP}

The study was performed in the Virtual Reality Eye Tracking Lab at Clemson University. The VR system is driven by a $1.5 \mathrm{GHz}$ dual processor PC running Red Hat Linux (v 8.0, kernel v2.4.20) equipped with $1 \mathrm{~Gb}$ RAM and an NVidia GeForce4 Ultra 5950 graphics card. The hardware components include, the binocular eye tracker mounted in Virtual Research V8 HMD and 6 DOF
Flock of Birds (Figure 1). Eye movements are tracked using a video base corneal reflection

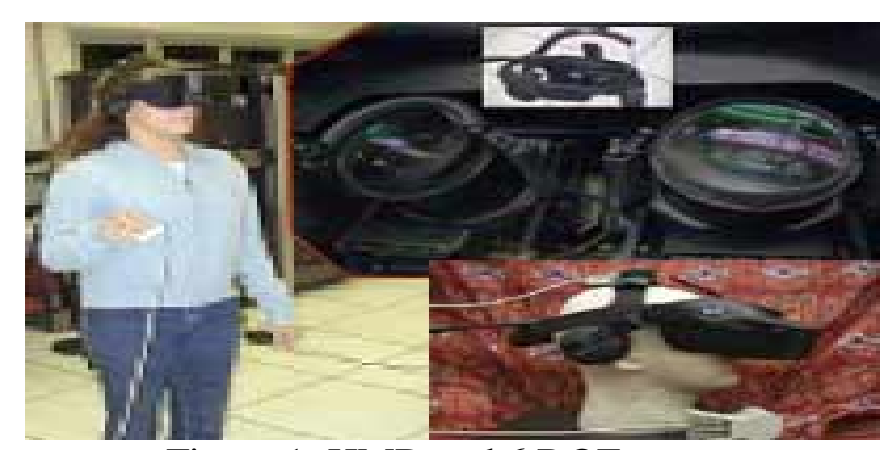

Figure 1. HMD and 6 DOF mouse

binocular eye-tracker from ISCAN mounted within the helmet. The frame rate of this VR system is 30 fps (frame per minute). Vspec, Inspect and Inspector were the software components used in this study, custom developed by Clemson University researchers. The Inspector program uses the $\mathrm{C}++$ /OpenGL language to display the VR scenario to the participants while recording their eye movements. Vspec performs offline analysis of the participant's point of regard (POR) data. The CVE is enabled by Inspect. No data is recorded in the CVE.

\section{STIMULUS}

Three inspection scenarios used in this study -2 task scenarios for the pre-test and posttest and 1 for CVE training session, were variations of a VR aft aircraft cargo bay model (Figure 2) similar to the cargo bay of L1101 aircraft. These inspection scenarios had similar difficulty levels in terms of the number and types of defects and their location. A total of 22 defects were placed in each scenario. The defect types were crack, corrosion, hole, abrasion and broken electrical conduit.

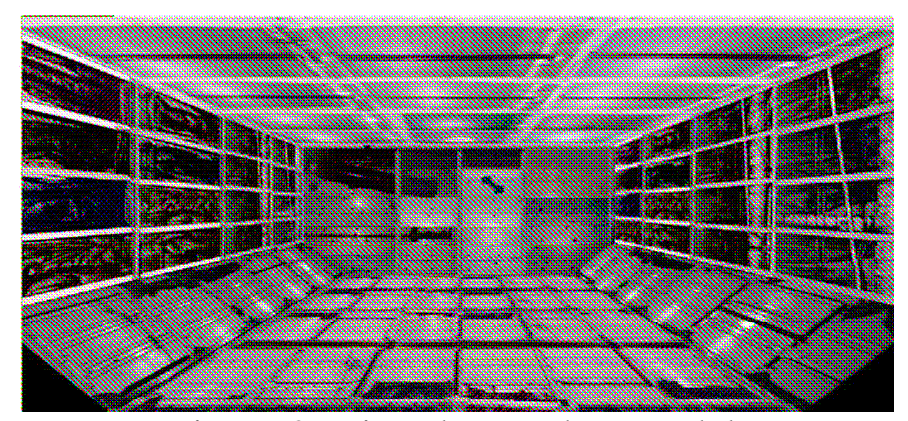

Figure 2. Virtual cargo bay model 


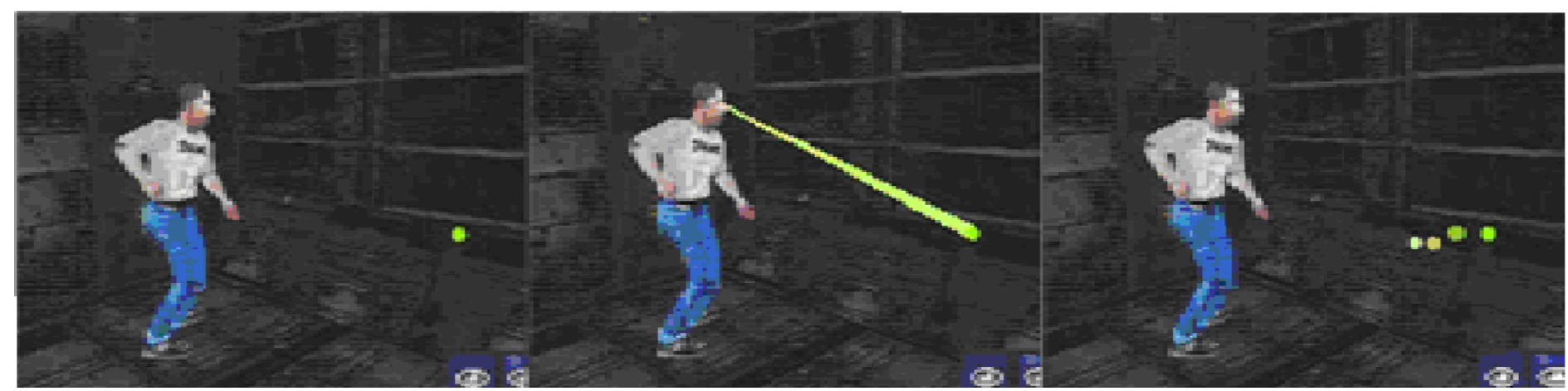

Figure 3. Three display techniques used to represent the gaze slaved deictic reference of the trainer

Three display techniques - a dot, ray casting and a decaying trace - used in this study provided a unique representation of the expert's search strategy to the trainee (Figure 3). The dot represented the instantaneous point of regard (POR) of the expert, similar to a laser pointer used in presentations. The ray casting, analogous to a flashlight display, provided the trainee with the source of origination of the ray. In this display form, a yellowish green semi-transparent cone-shaped ray of light slaved to the gaze orientation of the expert was used for communicating the search strategy to the trainee. This type of display enables the trainee to regain the expert's POR in case of disorientation. The decaying trace provides a brief positional history of the sequence of path followed by the expert during inspection. The decay time for the trace was set to $200 \mathrm{~ms}$. In this type of display form, yellowish green dots fade away into the environment at an interval of $200 \mathrm{msec}$. The trail of dots representing the expert's POR while performing inspection is perceived in the form of a transparent line moving continuously similar to a trace. Under dim lightning conditions, typically found in inspection booths, eye is most sensitive to yellowish green light with wavelength $550 \mathrm{~nm}$. Considering the medium gray background of the aircraft cargo bay with low levels of illumination, yellowish green color with an RGB setting of $(0.0,0.77,0.0)$ was used.

\section{PROCEDURE}

A pilot study was conducted to set the pacing condition for the inspection tasks to counteract any SATO effects. The pacing time was set at six minutes (one standard deviation below the mean). A 'Pre-test Post-test Control Group Design' was adopted with 32 participants enrolled at Clemson University randomly assigned 8 per group to three treatment groups (dot, ray casting and decaying trace) and one control group. The participants were screened for $20 / 20$ vision, color perception and assurance that none had prior experience with the VR. The participants were first immersed in a familiarization scenario with training on the defects provided followed by them performing an inspection task in one of the two multi-defect inspection scenarios. The inspection task required the participants to search for the defects and select them using a 6DOF mouse. If the selection was correct, the defect was highlighted in red. The participants in the treatment groups then underwent unpaced search strategy training in the CVE. Here, the participants were co-immersed with an expert performing an inspection task and they were required to follow and understand the expert's search strategy aided by the VDR displayed by one of the three methods depending on their group. The participants in the control group received no training. All the participants then performed an inspection task in the multi-defect inspection scenario different from the one, they were exposed to before. These inspection scenarios were counterbalanced to assure that same number of orderings for each group. Data was collected on the number of defects detected the performance measure and four process measures: number of fixation points, number of fixation groups, average fixation duration and percentage area covered. A questionnaire was used to collect subjective information such as usefulness and effectiveness of training and display used. 

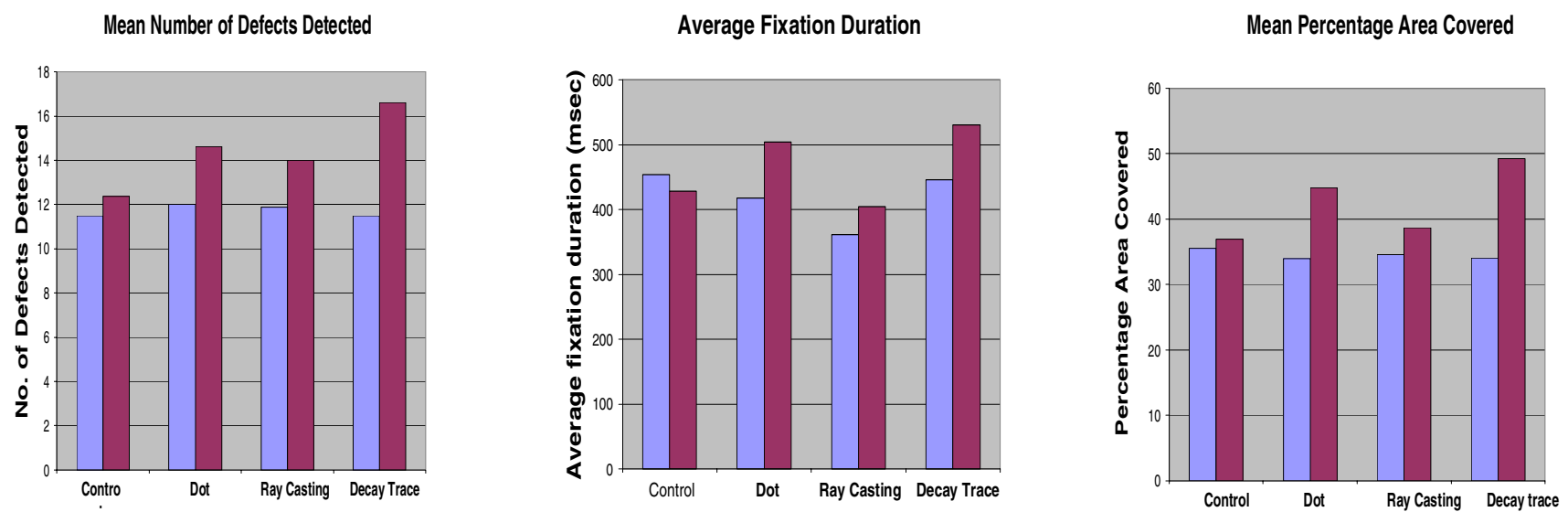

Pre-test Post-test

Figure 4. Graphical representation of the pre-test and post-test training data for the performance and process measures for the four conditions

\section{RESULTS AND DISCUSSION}

As seen in Figure 4, all the three treatment groups had higher means for the performance and process measures on their post training trials than the control group. However, in order to determine the improvement in the inspection performance of the participants in their post training trial, mean gain in the performance and process measures was calculated. The pre-test scores for each measure were identified as the covariate, as it was recognized that the participants with the lower pretest scores on a specific measure have the potential to achieve higher gain. Analysis of covariance (ANCOVA) was used to determine the main and interaction effects of the groups, comparing the gain in the performance and process measures, controlling for the effects of the pre-test scores. Gain was calculated as the difference (post-test pre-test) focusing on the improvement in the participant's abilities after training. An LSMEANS test was then performed to investigate the multiple pairwise comparisons of the mean gain for the performance and process measures for different participant groups. Subjective measures were analyzed using a Wilcoxon signed-rank test.

There was significant difference found in the gain in the number of defects detected between the participant groups $(\mathrm{p}<0.01)$. The three treatment groups had significantly higher mean gain in the number of defects detected than the control group, thus emphasizing the effectiveness of CVE training. The decaying trace had the highest mean gain among the treatment groups. While there was an increase in the number of fixation points and fixations groups after training, the difference between the groups was not significant. ( $p>0.05)$. The gain in the average fixation duration differed significantly between the participant groups $(\mathrm{p}<0.05)$, which showed that participants adopted a slower search strategy in their post-training trials to minimize chances of missing a defect. The decaying trace exhibited significantly higher mean gain in the average fixation duration than control and ray casting groups, but not significantly higher than the dot group. A significant difference in the gain in the percentage of area covered (Figure 5) between the participant groups $(\mathrm{p}<0.05)$ was observed. The decaying trace had significantly higher mean gain in the percentage area covered than the control and the ray casting groups but not significantly higher than the dot group. There was no significant interaction effect of the pre-test scores observed on any measure. This indicates that the effect of the pre-test scores on performance and process measures remains the same in all participant groups. The participants in the decay trace and the dot groups tended to adopt a much slower paced search strategy during their post-test inspection trail in order to cover the maximum area of the cargo bay efficiently without overlap. 

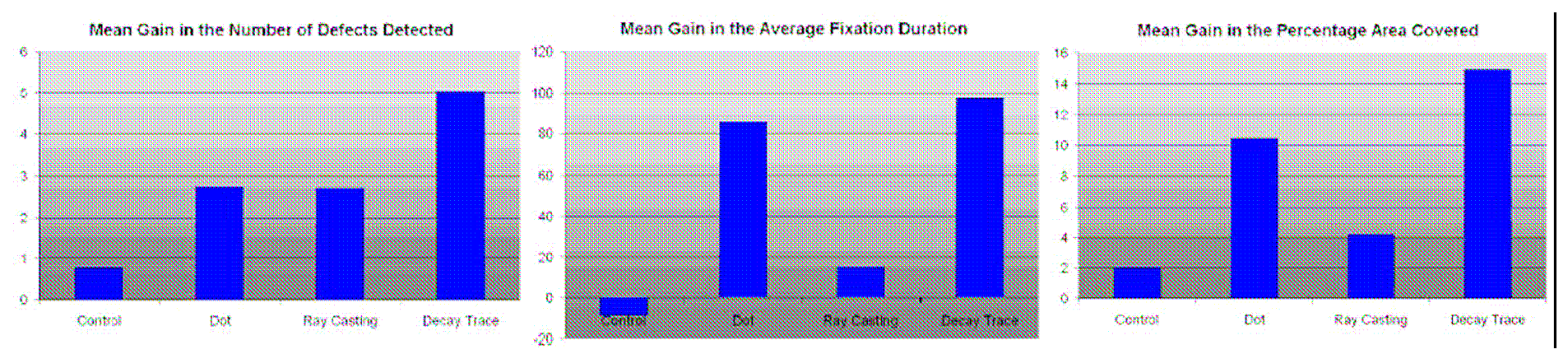

Figure 5. Graphical representation of the mean gain in performance and process measures for the four conditions

The lower mean gain on the average fixation duration and percentage area covered for ray casting group can be attributed to the fact that this type of display form failed to give an accurate representation of the sequence of the path taken by the expert. As a result the participants tended to still adopt a more random search strategy, which is faster and involves overlapping similar to the control. The subjective measures indicate that the participants perceived search strategy training using CVE to be effective and pointed out the decaying trace as the most effective display technique to represent the expert's deictic reference.

\section{CONCLUSION}

Thus to conclude, search strategy training in CVE aided by the visual representation of the expert's VDR is effective in improving novice inspector's inspection and search performance. The novice inspectors can be trained to adopt systematic search strategy. On basis of the analyses conducted on the performance, process and subjective measures, the decaying trace emerges as the most the effective display technique for representing and communicating the expert's search strategy. This has implications in visual search training in VR for aircraft inspection tasks.

\section{AKNOWLEDGEMENTS}

This work was supported in part by NSF ITR award \# 0217600. Avatar (MD3) model format courtesy of Id Software, Inc. We would like to acknowledge Nathan Cournia from the Department of Computer Science for the development of the collaborative virtual environment.

\section{SELECTED REFERENCES}

1. Duchowski, A., Shivashankariah,V., Rawls, T., Gramopadhye, A., Melloy, B., \& Kanki, B. (2000). Binocular Eye Tracking in Virtual Reality for Inspection Training. In Eye Tracking Research \& Applications (ETRA) Symposium, ACM, 89-96.

2. Hindmarsh, J., Fraser, M., Heath, C., Benford, S., \& Greenhalgh, C. (2000). Objectfocused interaction in collaborative virtual environments. In Transactions on ComputerHuman Interaction (TOCHI), 7(4), 477-509.

3. Vertegaal R. (1999). The GAZE groupware system: mediating joint attention in multiparty communication and collaboration. Proceedings of the SIGCHI conference on Human Factors in computing systems: $\mathrm{CHI}$ is the limit, Pittsburgh, PA, 294-301.

4. Kundel, H.L., Nodine, C.F., \& Krupinski, E.A. (1990). Computer-Displayed Eye Position as a Visual Aid to Pulmonary Nodule Interpretation. Investigative Radiology, 25(8), 890-898.

5. Drury, C.G., and Gramopadhye, A.K. (1990). Training for visual inspection. Paper presented at the Third FAA Conference on Human Factors in Aircraft Maintenance and Inspection: Training Issues. Atlantic City.

6. Benjamin Law, M. Stella Atkins, A. E. Kirkpatrick, Alan J, Lomax, "Eye gaze patterns differentiate novice and experts in a virtual laparoscopic surgery training environment", Proceedings of the Eye tracking research \& applications symposium on Eye tracking research \& applications, pp 41-48, March 22-24,2004 San Antonio, Texas. 